hep-th/9706164

IPM-97-208

March 1997

\title{
Exactly and Quasi-Exactly Solvable Models on the Basis of $\operatorname{osp}(2 \mid 1)$
}

\author{
A. Shafiekhani ${ }^{\dagger}$ " and M. Khorrami ${ }^{\dagger *}$ \& \\ ${ }^{\dagger}$ Institute for Studies in Theoretical Physics and Mathematics \\ P.O.Box: 5531, Tehran, 19395, Iran, \\ * Department of Physics, Tehran University, \\ North-Kargar Ave. Tehran, Iran. \\ and \\ Institute for Advanced Studies in Basic Sciences, \\ P.O.Box: 159, Gava Zang, Zanjan 45195, Iran.
}

\begin{abstract}
The exactly and quasi-exactly solvable problems for spin one-half in one dimension on the basis of a hidden dynamical symmetry algebra of Hamiltonian are discussed. We take the supergroup, $\operatorname{OSP}(2 \mid 1)$, as such a symmetry. A number of exactly solvable examples are considered and their spectrum are evaluated explicitly. Also, a class of quasi-exactly solvable problems on the basis of such a symmetry has been obtained.
\end{abstract}

\footnotetext{
${ }^{1}$ E-mail: ashafie@theory.ipm.ac.ir

$2 \quad$ mamwad@netware2.ipm.ac.ir
} 


\section{Introduction}

In the last few years, a new class of quantum mechanical problems has been investigated. This is the class of quasi-exactly solvable problems [1-6], which in some sense is an intermediate class between problems for which the spectrum can be found exactly, analytically or algebraically, and those which can not be solved. A hidden symmetry group with a finite dimensional space of wave functions is responsible for the existence of quasi-exactly solvable problems [3, 4]. Such a symmetry is inherent to any exactly and quasi-exactly solvable problem. In the exactly solvable problems case, the symmetry is, in some sense, complete, and the Hamiltonian can be diagonalized. In the case of quasi-exactly solvable models, the Hamiltonian is only block diagonalized, and there is a finite block which can be diagonalized. Therefore only part of the spectrum can be found. In both cases the Hamiltonian can be reduced to at most quadratic combinations of generators of a relevant symmetry group with finite dimensional representations. Such Hamiltonians can be written in standard form by choosing differential realizations of generators. Differential realizations of generators are representations of generators in a finite dimensional subspace of analytical functions [7]. Hence, one will be able to compute the spectrum by using these realizations. There have been attempts to do so for spinless [3, 4, 5] and spin one-half [5, 8-11] particles. In this paper the supergroup $O S P(2 \mid 1)$ has been taken as symmetry group of a spin one-half particle.

Differential realizations of the generators of this group contains beside $z$ and $\frac{\partial}{\partial z}$, $\theta$ and $\frac{\partial}{\partial \theta}$, where $\theta$ is a a Grassmann variable. Therefore the Hamiltonians are function of $z, \frac{\partial}{\partial z}, \theta$, and $\frac{\partial}{\partial \theta}$. To get the standard form of the Hamiltonian, we use proper change of variable from $z$ to $x$, take $x$ as the position variable, and put

$$
\theta:=\left(\begin{array}{cc}
0 & 0 \\
1 & 0
\end{array}\right), \quad \frac{\partial}{\partial \theta}:=\left(\begin{array}{ll}
0 & 1 \\
0 & 0
\end{array}\right) .
$$

Hence, we will have standard form of Hamiltonian with spin interaction terms.

Our aim in this paper is to give a new class of exactly and quasi-exactly solvable problems. The structure of this paper is as follows: In section 2, we construct general exactly solvable Hamiltonians for spin one-half particles on the basis of the supergroup $O S P(2 \mid 1)$, and find the corresponding spectrum. In section 3 we generalise the Hamiltonians given in section 2 to a class of quasi-exactly solvable problems for spin one-half particles.

\section{Exactly Solvable Models}

One of the major symmetry group candidates for spin one-half particles is the supergroup with rank one, $O S P(2 \mid 1)$. Such a group has three even and two odd generators. The 
differential realization of the algebra $\operatorname{osp}(2 \mid 1)$ is obtained in [5, 12]:

$$
\begin{array}{ccc}
J_{-}=\partial & =: & J_{-1} \\
J_{+}=-z^{2} \partial+2 h z-z \theta \delta & =: & J_{+1} \\
J=2 z \partial-2 h+\theta \delta & =: & J_{0} \\
G_{-}=\delta+\theta \partial & =: & J_{-\frac{1}{2}} \\
G_{+}=z \delta+\theta z \partial-2 h \theta & =: & J_{+\frac{1}{2}}
\end{array}
$$

where $\left\{J_{ \pm}, J_{0}\right\}$ are even and $\left\{G_{ \pm}\right\}$are odd generators, $\theta$ is a Grassmanian variable, $h$ is half of the spin of the representation, and $\partial=\frac{\partial}{\partial_{z}}, \delta=\frac{\partial}{\partial_{\theta}}$.

One can consider this, as the representation of $\operatorname{osp}(2 \mid 1)$ on the super-sub-space of analytic functions spanned by the monomials,

$$
\mathcal{F}_{h}:=\left\{z^{p} \theta^{q} \mid p=0,1, \cdots, 2 h, q=0,1\right\}
$$

These generators satisfy the (anti)commutation relations

$$
\begin{array}{ccc}
{\left[J_{+}, J_{-}\right]=J} & \left\{G_{ \pm}, G_{ \pm}\right\}=\mp 2 J_{ \pm} & \left\{G_{+}, G_{-}\right\}=J \\
{\left[J, J_{ \pm}\right]= \pm 2 J_{ \pm}} & {\left[J, G_{ \pm}\right]= \pm G_{ \pm}} & {\left[J_{+}, G_{-}\right]=G_{+}} \\
{\left[J_{+}, G_{+}\right]=0} & {\left[J_{-}, G_{+}\right]=G_{-}} & {\left[J_{-}, G_{-}\right]=0}
\end{array}
$$

The Casimir of this realizations is

$$
J_{0}^{2}+2\left\{J_{-}, J_{+}\right\}+\left[G_{-}, G_{+}\right]=2 h(2 h+1)
$$

To write a solvable Hamiltonian, we note that the generator $J_{\alpha}$ ( one can call $\alpha$ the spin) acting on $z^{p} \theta^{q}$, changes the index $p+\frac{q}{2}$ to $p+\frac{q}{2}+\alpha$. Moreover, the set of generators (2) acting on states with $p+\frac{q}{2} \geq 0$, give rise to states with $p+\frac{q}{2} \geq 0$. Hence, one can regard the state $z^{p=0} \theta^{q=0}$ as the ground state. Now, any combination of operators with total $\alpha$ not greater than zero has a matrix representation which is upper triangular, where the ordering of the states $z^{p} \theta^{q}$ is according to $p+\frac{q}{2}$. The eigenvalues of such a (n infinite dimensional) matrix are its diagonal elements and the eigenvectors can be obtained recursively.

Hence, to write a solvable Hamiltonian it is sufficient to add terms of the type $J_{\alpha_{1}} \cdots J_{\alpha_{n}}$, where $\sum \alpha_{i} \leq 0$. As we want to convert such a Hamiltonian to a Schrödinger one, that is one with a second order derivative and a potential, we must keep only terms at most quadratic in the generators. Moreover, (5) shows that not all second order generators are independent. 
We can list all up to second order independent spin-non-positive generators.

$$
\begin{aligned}
& \operatorname{spin}-2 \quad: \quad A:=\partial^{2}=J_{-}^{2} \\
& \operatorname{spin}-3 / 2: \partial \delta+\partial^{2} \theta=\frac{1}{2}\left\{J_{-}, G_{+}\right\} \\
& \text {spin }-1:\left\{\begin{array}{l}
B:=\partial=J_{-} \\
C:=4 z \partial^{2}+2 \partial \theta \delta=\left\{J, J_{-}\right\}+(4 h-2) J_{-}
\end{array}\right. \\
& \operatorname{spin}-1 / 2:\left\{\begin{array}{l}
\delta=\frac{1}{2(1+4 h)}\left[(1+4 h) G_{-}+2\left\{J_{-} G_{+}\right\}-\left\{J, G_{-}\right\}\right] \\
\theta \delta=\frac{1}{2(1+4 h)}\left[(1+4 h) G_{-}-2\left\{J_{-}, G_{+}\right\}+\left\{J, G_{-}\right\}\right] \\
z \partial \delta+z \partial^{2} \theta=\frac{2 h-1}{2} G_{-}+\frac{1}{2(1+4 h)}\left[\left\{J_{-}, G_{+}\right\}+2 h\left\{J, G_{-}\right\}\right]
\end{array}\right. \\
& \operatorname{spin} 0:\left\{\begin{array}{r}
D:=z \partial=\frac{1}{2}(J+2 h)-\frac{1}{2(1+4 h)}\left(\left[G_{-}, G_{+}\right]+2 h\right) \\
E:=\theta \delta=\frac{1}{1+4 h}\left(\left[G_{-}, G_{+}\right]+2 h\right) \\
F:=z^{2} \partial^{2}+z \partial \theta \delta=\frac{1}{4}\left[(J+2 h)^{2}-2 J-4 h\right] \\
\quad+\frac{1}{4(1+4 h)}\left(\left[G_{-}, G_{+}\right]+2 h\right)
\end{array}\right.
\end{aligned}
$$

Another thing to be considered is that we want to use this combination and then make a change of variable $z \rightarrow x$ and a quasi gauge transformation to bring the Hamiltonian to a standard form. Now, as beside $z$ there exist $\theta$ and $\delta$ in Hamiltonian, this change of variable depends on $\theta$ and $\delta$, which shows that $x$ no longer commutes with $\theta$ and $\delta$. However, if only the combination $\theta \delta$ appears in the Hamiltonian, $x$ will contain only $z$ and $\theta \delta$, and the final Hamiltonian will consist of just $x$ and $\theta \delta$. In this case, although $x$ does not commute with $\theta$ and $\delta$, it does commute with $\theta \delta$ and the Hamiltonian has its standard form: having a space degree of freedom and some internal degree with a combination of this parameter which commutes with the space degree of freedom.

So, the Hamiltonian which we consider is of the form

$$
\tilde{H}=a A+b B+c C+d D+e E+f F .
$$

This Hamiltonian acts on the space spanned by the basis $\left\{z^{p} \theta^{q}\right\}$, where $p$ is nonnegative and integer, and $q=0,1 . \mathcal{F}_{h}$ is a subspace of this space.

As it is shown below, one can use the variable $x$, instead of $z$ and make a quasi gauge transformation to make $H$ in the form

$$
H=-\frac{\hbar^{2}}{2 m} \frac{d^{2}}{d x^{2}}+V(x, q)
$$

Here one can interpret $x$ as the space coordinate and $q$ as the matrix $\left(\begin{array}{ll}1 & 0 \\ 0 & 0\end{array}\right)$. Then $H$ is a Hamiltonian of an spin 1/2 system, and obviously Hermitian with respect to the inner product $\langle f, g\rangle:=\int f^{\dagger}(x) g(x) d x$, where $f$ and $g$ are functions $\mathcal{R} \rightarrow \mathbb{C}^{2}$. 


\subsection{Explicit Examples}

The Hamiltonian (7) can be written in the form

$$
\tilde{H}=\left(f z^{2}+4 c z+a\right) \frac{\partial^{2}}{\partial z^{2}}+[(b+2 c q)+(d+f q) z] \frac{\partial}{\partial z}+e q
$$

where

$$
q:=\theta \delta
$$

The spectrum of this Hamiltonian is simply the diagonal elements of it in $z^{p} \theta^{q}$ representation, that is

$$
E_{p, q}=f p[(p-1)+q]+d p+e q .
$$

Now consider the following cases.

i) $c=f=0 \neq a$

In this case, (9) becomes

$$
\tilde{H}=a\left[\frac{\partial}{\partial z}+\frac{b+d z}{2 a}\right]^{2}-\frac{(b+d z)^{2}}{4 a}-\frac{\partial}{2}+e q
$$

and, by a simple change of variable and a pseudo-gauge transformation 四, 鴫, to eliminate the first order derivative,

$$
H=a \frac{\partial^{2}}{\partial x^{2}}-\frac{d^{2}}{4 a} x^{2}-\frac{d}{2}+e q .
$$

Using

$$
\left\{\begin{array}{l}
a=-\frac{\hbar}{2 m} \\
d=\hbar w
\end{array}\right.
$$

it is seen that (13) is the Hamiltonian of a Harmonic oscillator which is also confined in a magnetic field. The spectrum of (13) is

$$
E_{p, q}=d p+e q
$$

ii) $f=0 \neq c$

The Hamiltonian becomes

$$
\tilde{H}=(a+4 c z) \frac{\partial^{2}}{\partial z^{2}}+(b+d z) \frac{\partial}{\partial z}+2 c q \frac{\partial}{\partial z}+e q .
$$

Using

$$
x=\sqrt{z+\frac{a}{4 c}}
$$

and a proper pseudo-gauge transformation, we arrive at

$$
H=c \frac{\partial^{2}}{\partial x^{2}}-\frac{d^{2}}{16 c} x^{2}-\frac{d}{4}-\frac{c \zeta(1+\zeta)}{x^{2}}+\frac{d \zeta}{2}+e q
$$


where

$$
\zeta:=\frac{2 c-2 c q-b+b a /(4 c)}{4 c}
$$

Now, setting

$$
\left\{\begin{array}{l}
c=-\frac{\hbar^{2}}{2 m} \\
d=2 \hbar \omega
\end{array}\right.
$$

it is seen that $H$ is very similar to the radial part of the Hamiltonian of an isotropic Harmonic oscillator in a magnetic field. There are, however, nontrivial interactions; the terms $\frac{q}{x^{2}}$ and $\frac{q^{2}}{x^{2}}$, which represent spin-nonuniform magnetic field interactions. The spectrum of this Hamiltonian is

$$
E_{p, q}=d p+e q
$$

iii) $f \neq 0$

By a suitable shift in $z$, (9) becomes

$$
\tilde{H}=\left(a+f z^{2}\right) \frac{\partial^{2}}{\partial z^{2}}+[b+(d+f q) z] \frac{\partial}{\partial z}+e q .
$$

Then, using

$$
z=\sqrt{\frac{a}{f}} \sinh x,
$$

and a proper pseudo-gauge transformation,

$$
\begin{aligned}
H & =-f\left[-\frac{\partial^{2}}{\partial x^{2}}+\frac{(q-1+d / f)^{2}}{4} \tanh ^{2} x+\left(\frac{b^{2}}{4 a f}+\frac{q-1+d / f}{2}\right) \cosh ^{-2} x\right. \\
& +\frac{b}{2 \sqrt{a f}}\left(2-q-\frac{d}{f}\right) \frac{\sinh x}{\cosh ^{2} x}+e q .
\end{aligned}
$$

The spectrum of this is

$$
E_{p, q}=f p(p-1)+f p q+d p+e q .
$$

Note that all of the Hamiltonians so obtained, are Hermitian.

\section{Quasi-Exactly Solvable Problems}

Up to now, we investigated only the exactly solvable problems. To go beyond, and investigate quasi-exactly solvable problems, we should also consider the spin-positive generators.

$$
\begin{aligned}
\operatorname{spin} 2 & : A^{\prime}:=z^{4} \partial^{2}-2 k z^{3} \partial+2 h k z^{2}+\left[2 z^{3} \partial-2 k z^{2}\right] \theta \delta=J_{+}^{2} \\
\operatorname{spin} 3 / 2: & {\left[-z^{3} \partial+k z^{2}\right] \delta+\left[-z^{3} \partial^{2}+2 k z^{2}-2 h k z\right] \theta=\frac{1}{2}\left\{J_{+}, G_{+}\right\} } \\
\operatorname{spin} 1 & :\left\{\begin{array}{l}
B^{\prime}:=-z^{2} \partial+2 h z-z \theta \delta=J_{+} \\
C^{\prime}:=-2 z^{3} \partial^{2}+2 k z^{2} \partial+\left[-3 z^{2} \partial+k z\right] \theta \delta=\frac{1}{2}\left\{J, J_{+}\right\}+k J_{+\frac{1}{2}}
\end{array}\right.
\end{aligned}
$$

where $2 h-1=k$. By the same reason of section 2, the Hamiltonian should consist of just $z$ and $\theta \delta$, so final Hamiltonian which we consider for quasi-exactly solvable problem is of the form

$$
\tilde{H}=a A+a^{\prime} A^{\prime}+b B+b^{\prime} B^{\prime}+c C+c^{\prime} C^{\prime}+d D+e E+f F .
$$


The above Hamiltonian can be written in form of

$$
\begin{aligned}
\tilde{H} & =\left(a^{\prime} z^{4}-2 c^{\prime} z^{3}+f z^{2}+4 c z+a\right) \partial^{2}+\left[2 a^{\prime}(q-k) z^{3}+\left((2 k-3 q) c^{\prime}-b^{\prime}\right) z^{2}\right. \\
& +(d+f q) z+(b+2 c q)] \partial+2 a^{\prime} k(h-q) z^{2}+\left[c^{\prime} k q+b^{\prime}(2 h-q)\right] z+e q \\
& :=\mathcal{P}_{4}(z) \partial^{2}+\mathcal{P}_{3}(z, h, q) \partial+\mathcal{P}_{2}(z, h, q) .
\end{aligned}
$$

In the above expression for Hamiltonian $h$ plays an important role for quasi-exactly solvable problems. The Hamiltonian matrix is in block-diagonal form with two blocks: one is $2 h+1$ dimensional and the other is infinite-dimensional. The former block can be diagonalized, as it is finite dimensional.

To bring the Hamiltonian to the standard form, the following change of variable should be done

$$
x=\alpha \int \frac{d z}{\sqrt{\mathcal{P}_{4}(z)}} .
$$

Using the above change of variable the Hamiltonian will be

$$
\tilde{H}=\alpha \frac{\partial^{2}}{\partial x^{2}}+2 \alpha \mathcal{A}(x, q, h) \frac{\partial}{\partial x}+\mathcal{P}_{2}(x, q, h)
$$

where

$$
\mathcal{A}(x, q, h)=\left.\frac{1}{4 \alpha}\left[\frac{2 \mathcal{P}_{3}(z, q, h)-\mathcal{P}_{4}^{\prime}(z)}{\sqrt{\mathcal{P}_{4}(z)}}\right]\right|_{z=z(x)}, \quad \mathcal{A}=\frac{\partial a(x)}{\partial x} .
$$

In the above expression $a(x)$ is pseudo-gauge, and potential is

$$
V(x, q, h)=\mathcal{P}_{2}(x, q, h)-\alpha\left(\frac{\partial \mathcal{A}}{\partial x}-\mathcal{A}^{2}\right) .
$$

Putting all together we have

$$
\tilde{H} \hat{\psi}=E \hat{\psi}
$$

where

$$
\psi=e^{-a(x)} \hat{\psi}, \quad\{\hat{\psi}\}=\mathcal{F}_{h} \cup\left\{\psi_{2 h+2}, \psi_{2 h+2} \theta, \cdots\right\} .
$$

In this way, one gets a Hamiltonian which is a similarity transformation of a block diagonal operator, having a finite block (corresponding to $\mathcal{F}_{h}$ ). So, the eigenvalues (and eigenvectors) corresponding to this space can be obtained.

\section{References}

[1] M. Razavy, Amer. J. Phys. 48 (1980) 285; Phys. Lett. 82A (1981) 7.

[2] A. Turbiner and A.G. Ushveridze, Phys. Lett. A 126(1987) 181.

[3] A. Turbiner, Commun. Math. Phys. 118 (1988) 467.

[4] M.A. Shifman, I.J.M.P. A4 (1989) 2897.

[5] M.A. Shifman and A. Turbiner, Commun. Math. Phys. 126 (1989) 347. 
[6] A. Minzoni, M. Rosenbaum and A. Turbiner, "Quasi-Exactly-Solvable Many-Body Problems", ICN preprint, ICN-UNAM 96-07, hep-th/9606092.

[7] A. Shafiekhani, Mod. Phy. Lett. A9 (1994) 3273.

[8] F. Finkel, A. Gonález-López and M.A. Rodríguez, "Quasi-Exactly-Solvable Spin 1/2 Schrödinger Operators", hep-th/9509057.

[9] Y. Brihyae and P. Kosinski, JMP 35 (1994) 3089.

[10] A. Turbinar, AMS Contem. Math. 160 (1994) 263.

[11] A. Gangopadhyaya, A. Khare and U.P. Sukhatme, "Methods for generating QuasiExactly Solvable Potentials," Unvi. of Chicago preprint UICHEP-TH/95-5, [hepth/950822].

[12] W-S. Chung and A. Shafiekhani, Phys. Lett. B381 (1996) 68. 